\title{
Imaging Internal Flows in a Drying Sessile Polymer Dispersion Drop using Spectral Radar Optical Coherence Tomography (SR-OCT)
}

\author{
Selin Manukyan a,*, Hans M. Sauer ${ }^{\mathrm{b}}$, Ilia V. Roisman ${ }^{\mathrm{a}}$, Kyle A. Baldwin ${ }^{\mathrm{c}}$, David J. Fairhurst ${ }^{\mathrm{c}}$, \\ Haida Liang ${ }^{\mathrm{c}}$, Joachim Venzmer ${ }^{\mathrm{d}}$, Cameron Tropea ${ }^{\mathrm{a}}$ \\ ${ }^{a}$ Technische Universität Darmstadt, Institute of Fluid Mechanics and Aerodynamics, Petersenstr. 30 and Center of \\ Smart Interfaces, Petersenstr. 32, 64287 Darmstadt, Germany \\ ${ }^{b}$ Technische Universität Darmstadt, Institute of Printing Science and Technology, Magdalenenstr. 2, 64289 Darmstadt, \\ Germany \\ ${ }^{c}$ School of Science $\mathcal{E}$ Technology, Nottingham Trent University, Clifton Lane, Nottingham NG11 8NS, UK \\ ${ }^{d}$ Evonik Industries AG, Goldschmidtstr. 100, 45127 Essen, Germany
}

\begin{abstract}
In this work, we present the visualization of the internal flows in a drying sessile polymer dispersion drop on hydrophilic and hydrophobic surfaces with Spectral Radar Optical Coherence Tomography (SR-OCT). We have found that surface features such as the initial contact angle and pinning of the contact line, play a crucial role on the flow direction and final shape of the dried drop. Moreover, imaging through selection of vertical slices using optical coherence tomography offers a feasible alternative compared to imaging through selection of narrow horizontal slices using confocal microscopy for turbid, barely transparent fluids.
\end{abstract}

Keywords: OCT, polymer dispersion, internal flows

\section{Introduction}

In the area of paints and coatings, polymer coatings are often applied as an aqueous dispersion, which is obtained by emulsion polymerization. Such polymer dispersions consist of soft polymer particles surrounded by a water phase. After drying, the polymer particles merge, forming a homogeneous polymer film. The drying process of polymer dispersions is a very comprehensive topic. Due to their complex chemical properties, polymer dispersions show unexpected drying phenomena and intriguing final structure; one possible defect is the orange peel finish. These final structures play a crucial role in coating, printing and chemical drying processes. Despite a perfectly smooth substrate, during coating/painting processes the paint/coating might give a rough, orange skin looking finishing which could conceivably be the cause of future corrosion, aerodynamical instability or peeling off of the coating.

Drop desiccation and particle accumulation in sessile droplets has been frequently reported on in the literature. Deegan et al. [1, 2, 3] observed and explained how outward radial flows in

*Corresponding author

Email address: manukyan@csi.tu-darmstadt.de (Selin Manukyan)

Preprint submitted to Journal of Colloid and Interface Science

July 19, 2012 
an evaporating droplet lead to suspended particles being preferentially deposited at the droplet perimeter, forming the common coffee-ring stain. They showed that two crucial droplet parameters are required to induce this outward flow: a pinned contact line and an enhanced evaporation rate at the droplet perimeter. In their work, they introduce a numerical model which is confirmed by the experiments of Morozumi et al. [4] and Park et al. [5] using very small $(\sim 50 \mu \mathrm{m})$ droplets, similar to those used in ink jet printing. Fukai et al. [6] investigated the evaporation process of water-xylene-polystrene droplets and concluded that the pinning time or the receding distance are important factors to determine the shape and dimension of the final shape. Moreover the results of $\mathrm{Hu}$ and Larson [7] revealed that the coffee-ring phenomena not only requires a high evaporation rate near the pinned contact line but also suppression of Marangoni flow.

Pauchard and Allain $[8,9]$ used various concentrations of the Dextran solution to vary the liquid rheology and the initial contact angle and observed the effects of initial concentration, contact angle and relative humidity on the final structures formed during drying of polymer solution droplets. They identified several distinguishing final formations after desiccation including "Mexican hat" and "pancake" structures. The explanation for these structures is that as polymer concentration at the surface increases, a "glassy-skin" forms, which does not impede evaporation, and buckles under pressure induced by further evaporation from the bulk of the droplet. Ristenpart et al. [10] showed the change of circulation and magnitude in a sessile drop depending on the liquid-substrate thermal conductivity ratio. Moreover Popov [11], Head [12], Bhardwaj et al. $[13,14]$ and Maki et al. [15] introduced numerical and mathematical modelings on evaporation, particle accumulation and buckling of sessile drops on plane surfaces. Moreover Bhardwaj et al. $[13,14]$ and Maki et al. [15] expressed the surface tension driven Marangoni recirculation and skin formation due to weak diffusion in their experimental works.

Bodiguel et al. [16], Poulard et al. [17], Marin [18] and Nikolov et al. [19] visualized the particle accumulation by using interferometric methods and horizontal imaging. Kang et al. [20] and Jaijus et al. [21] applied PIV to visualize the flow direction in sessile drops of ethanol-water mixtures.

With the present work we show that the wetting features have a very important influence on the desiccation process of polymer dispersion drops. The final shape of the drop after drying is determined by the flow pattern inside the drop. Contact line pinning, initial contact angle and skin formation altogether influence the final dried shape of the sessile polymer drop. We succeeded to visualize this internal flow by scanning the cross section of the drop at its symmetry plane using Spectral Radar Optical Coherence Tomography (SR-OCT). Scanning the vertical cross section of targets is a novel method alternative to the usual confocal microscopy imaging.

OCT is a non-invasive imaging technique based on an imaging Michelson interferometer. There are broadly speaking two types of OCT: Time-domain and Fourier-domain OCT. In a Time-domain OCT, axial structure is obtained by scanning the reference mirror in the Michelson interferometer to change the reference path length. In a Fourier-domain instrument, the reference mirror is fixed but the interference fringes as a function of wavelength are obtained through a spectrometer and the axial structure is obtained through a Fourier transform [22]. OCT was invented for in vivo imaging of the eye and most applications are found in the biomedical field, but recently it has also found applications in non-biomedical fields [23] including the monitoring of the drying of thin films of various varnishes [24]. 


\section{Experiments}

Here we visualize the internal flows during the drying process of a sessile dispersion drop. We perform the measurements with a commercial Thorlabs SR-OCT, which enables observation inside the turbid liquid by scanning the drop cross sections vertically.

\subsection{Polymer Dispersion}

For the experiments, a pigment-free model paint provided by Evonik Industries AG has been used. This model paint was based on a polymer dispersion (NeoCryl XK232 / DSM Neoresins), combined with thickeners to match the rheological properties of a typical paint formulation. (The exact composition is given in the supporting information.) The particle size in the dispersion is $<1 \mu \mathrm{m}$. The paint formulation shows a shear thinning rheology, which is lost as the paint is diluted with water. The reference density of the model paint formulation diluted $50 \mathrm{vol}-\%$ with water is $1.0337 \mathrm{~g} / \mathrm{cm}^{3}$, the surface tension is $42 \mathrm{mN} / \mathrm{m}$ and the viscosity is $5 \mathrm{mPa} . \mathrm{s}$ at $100 \mathrm{1} / \mathrm{s}$. For the experiments the model paint has been diluted with distilled water; $10 \%-90 \%, 30 \%$ $70 \%$ and $50 \%-50 \%$ water/model paint mixtures were prepared. As substrates, clean borosilicate microscope slides (as hydrophilic surface - $100 \mu \mathrm{m}$ substrate thickness) and glass slides coated with Tegotop ${ }^{\circledR} 210$ (Evonik Industries AG) (as hydrophobic surface - $1 \mathrm{~mm}$ substrate thickness $+20 \mathrm{~nm}$ coating thickness) were used. All experiments were carried out in an air-conditioned environment at constant humidity $(35 \% \mathrm{Rh})$ and temperature $\left(22^{\circ} \mathrm{C}\right)$.

\subsection{Visualization Method}

To visualize the flows in the drop we used a Thorlabs SR-OCT, a Fourier domain OCT, which combines a broadband low coherence super-luminescent diode light source with a Michelson interferometer, a high speed spectrometer and a fast piezo-electric scanning mirror that enables a set of neighboring axial profiles to be collected to form a cross-section [25] (Figure 1). This system is ideal for imaging turbid samples and provides a direct measurement of scattered light along a vertical axis within a bulk sample. Fourier domain measurements enable each subsurface axial profile to be collected at the same time without time delay between the top and the bottom interface of a profile, which makes this technique very useful for internal flow observations. The backscattered light from various depths of the sample interferes with the light reflected from the reference mirror to produce an interference pattern which is measure by a spectrometer. Fourier transform of this interference fringe as a function of wavenumber gives the depth profile. The central wavelength of the light is $930 \mathrm{~nm}$ and the spectral bandwidth (FWHM) is $100 \mathrm{~nm}$. The imaging for our experiments is done at $2 \mathrm{fps}$ with an axial resolution of $7 \mu \mathrm{m}$ and lateral resolution of $9 \mu \mathrm{m}$. The maximum image depth and width are $1.6 \mathrm{~mm}$ and $4 \mathrm{~mm}$ respectively. The dilutions are mixed with tracer particles (copolymer microspheres-ThermoScientific) $4.3 \mu \mathrm{m}$ and $7.9 \mu \mathrm{m}$ in size.

Figure 2 schematically shows the different scanning procedures of confocal microscope and OCT. Confocal microscope select horizontal slices of the droplet sequentially while changing the focal plane by shifting the position of the pin hole. In case of OCT imaging, the 1D vertical (z-axis) profiles are obtained without physical scanning and the horizontal scanning is done by the movement of the scanning mirror, which shifts the ray along the direction perpendicular to the $\mathrm{x}-\mathrm{z}$ plane on the drop each time; with the help of a motorized table the substrate is moved in the $\mathrm{x}$-axis direction (direction perpendicular to the slice) to gain a 3-D image. The advantage of OCT is to be able to observe the cross section of the whole droplet on one frame. 


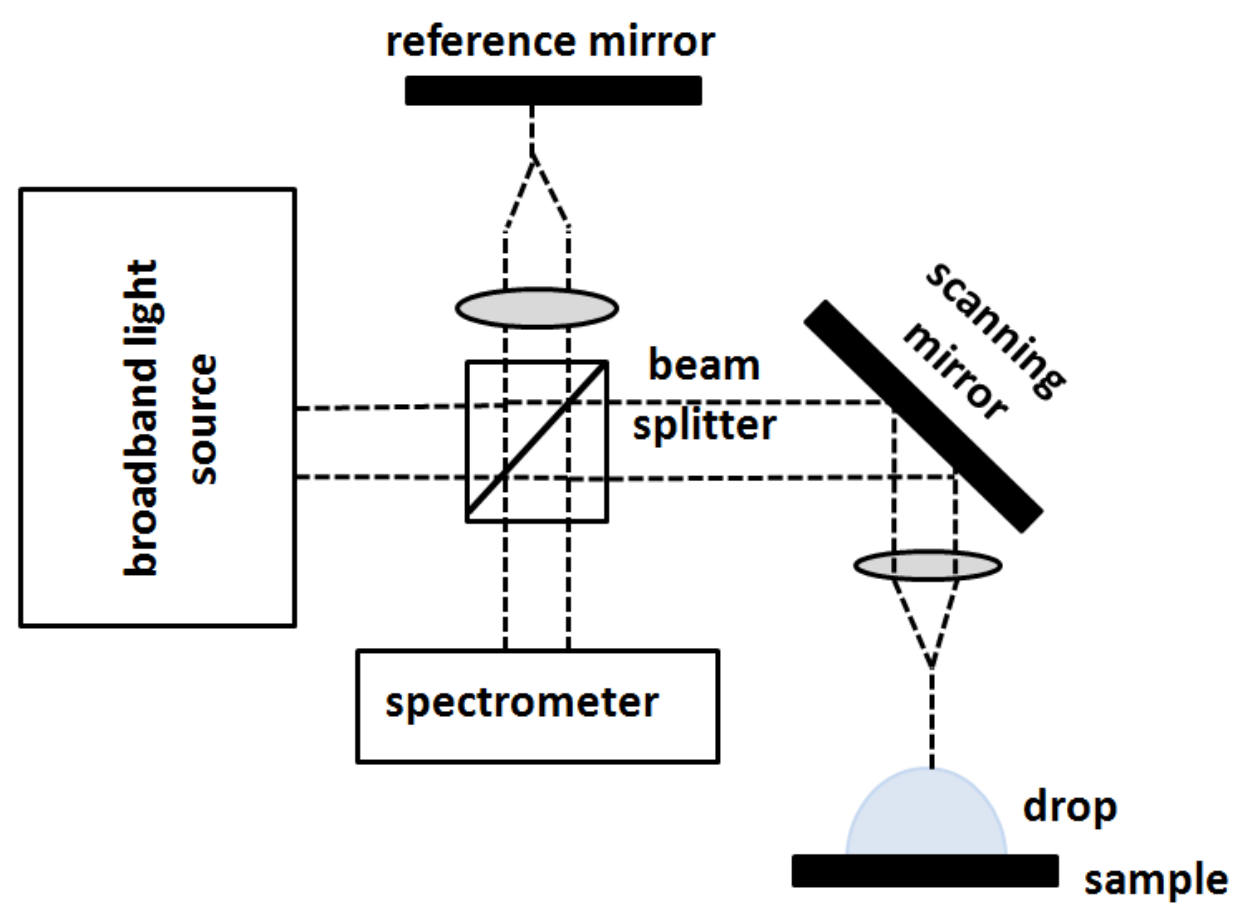

Figure 1: Schematic diagram of SR-OCT system.
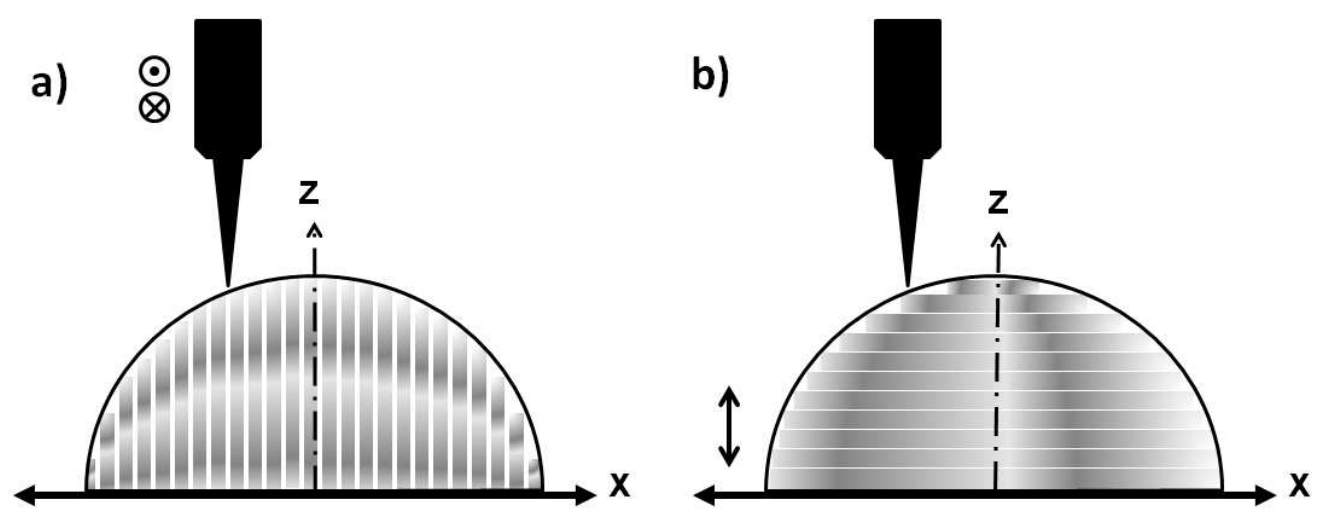

Figure 2: Comparison of scanning procedure of OCT (a) and confocal microscope (b)

\subsection{Experimental Procedure}

A sessile droplet of size 4.7-8.4 $\mu \mathrm{l}$ is placed on the target substrate and the internal flow circulations are observed by the cross-sectional particle tracking with OCT as the drop desiccated on the relaxation surfaces. The recording time (drying time) for a sessile droplet on a hydrophilic surface is between 10-15 minutes depending on the droplet volume and concentration. For a ses- 
sile droplet drying on a hydrophobic surface, the recording and drying time increases to 18-25 minutes under normal conditions.

\section{Results}

In our experiments a drying of a complex stationary droplet on a smooth rigid substrate at normal isothermal conditions is observed. Sessile polymer dispersion droplets start to desiccate from outside to inside and they build a skin as an outer shell during drying. We tested this formation by pricking a needle through the drop during desiccation. The drop reacted like a pimple, where the liquid paint trickled out of the hole. The skin formation does not appear to hinder the evaporation of the solvent (here water). Pinning of the contact line makes the skin formation more stable and affects the final shape of the drying drop. Polymer drops of size 4.7$8.4 \mu \mathrm{l}$ are left to dry on hydrophilic and hydrophobic surfaces to change the initial contact angle. With respect to the initial contact angle, the final shape becomes either a peak or an inversion of curvature (a bagel), leading to a collapse on the axis of droplet (see Figure 3).

hydrophilic substrate

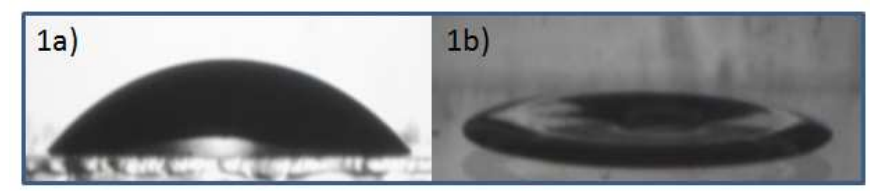

hydrophobic substrate

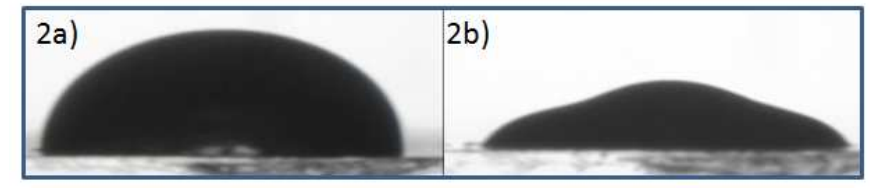

Figure 3: Drying process of 50\% model paint-50\% distilled water mixture before and after spreading: on hydrophilic surface (upper images) and hydrophobic surface (lower images). Initial and final shapes of the drop are shown for both cases (CCD camera images).

\subsection{Desiccation on hydrophilic surface $\left(\theta<45^{\circ}\right)$}

On a hydrophilic ${ }^{1}$ surface the three-phase contact line of the sessile dispersion droplet is pinned. This keeps the drop radius constant, leading to a gradual decrease in contact angle as the drop dries (see Fig. 4a). As the contact angle decreases, the enhanced evaporation rate close to the contact line leads to the polymer particles accumulate at the contact line, as described by Deegan et al.. Furthermore, as well as build up at the contact line, the rheological properties also change near this surface due to the local increase in particle concentration (see Fig. 5). If the drop dries slowly, a rigid transparent shell is formed at the surface. This shell does not prevent evaporation, so that the enclosed volume decreases while the contact line remains constant. During the desiccation process, the apex height first decreases, compressing the surface as it flattens. At the end of drying process there occurs a buckling in the center which has a form of "bagel" from top view.

\footnotetext{
${ }^{1}$ Here we define $\theta^{\circ}<45^{\circ}$ as hydrophilic, $45^{\circ} \leq \theta^{\circ} \leq 90^{\circ}$ as transition and $90^{\circ}<\theta^{\circ}<140^{\circ}$ as hydrophobic surface. The observations on $45^{\circ} \leq \theta^{\circ} \leq 90^{\circ}$ transition surface is not covered in this paper.
} 

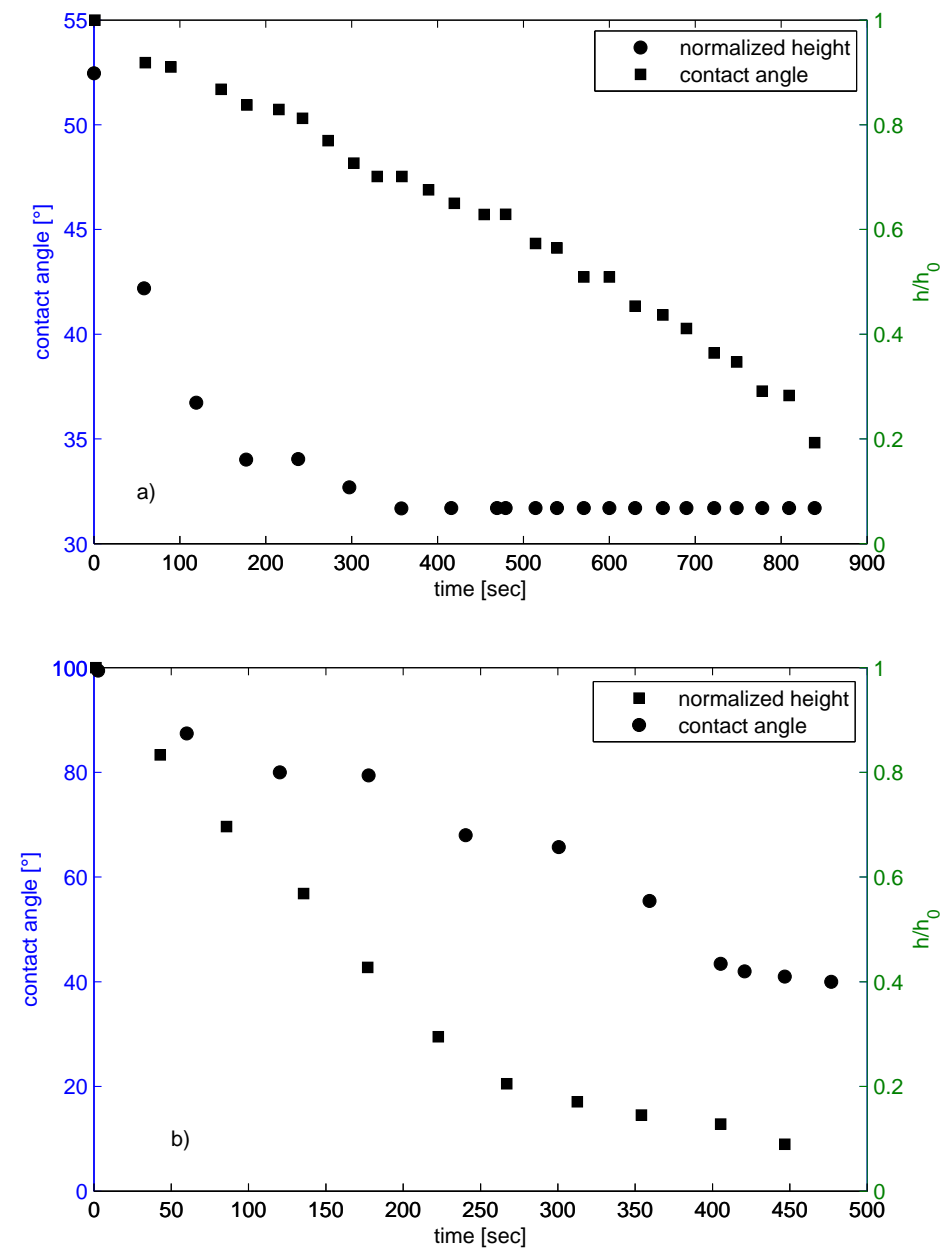

Figure 4: Drop height in the center of the drop and contact angle change vs. time for 50\% polymer dispersion-50\% distilled water mixture on hydrophilic (a) and hydrophobic (b) surfaces (data obtained from CCD camera images).

\subsection{Desiccation on hydrophobic surface $\left(90^{\circ}<\theta<140^{\circ}\right)$}

On a hydrophobic surface the three-phase contact line of the sessile dispersion droplet is also pinned, which keeps the drop radius constant (see Fig. 4b). Due to the large contact angle, the contact line radius has a smaller value compared to the sessile droplet on a hydrophilic surface, and the evaporation rate profile increases from bottom to top. The solid transparent shell evolves again, but this time has two distinctively different aspects to the hydrophilic case: the contact angle is increased, when the droplet reaches the glassy transition concentration; the lack of an enhanced evaporation rate at the contact line means that the outward radial flow dragging the particles to the contact line is reduced. This means that when the glassy-skin forms, it is much more uniform in thickness. The height of the drop decreases gradually due to water evaporation, but then, at a certain time, the contact angle and the height of the drop remain constant. At the 


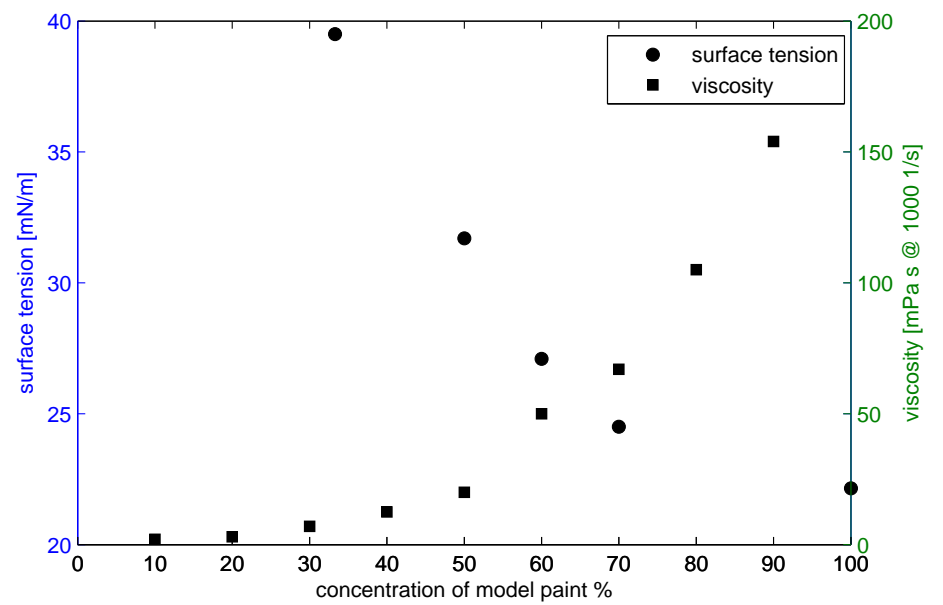

Figure 5: Surface tension and viscosity change depending on the concentration of the model paint. The surface tension decreases and the viscosity increases with increasing model paint concentration.

end there appears a ridge at the center. From OCT images it has also been observed that the evaporation process causes a cavity in the middle of the drop. The calculated refractive index $\left(\mathrm{n}_{\text {cavity }} \simeq 1\right)$ for the cavity is consistent with water vapour.

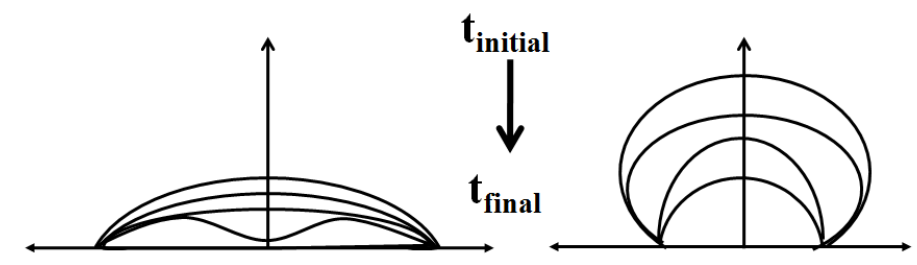

\section{a) hydrophilic surface b) hydrophobic surface}

Figure 6: Schematic representation of final shapes of the dying polymer drops on two different surfaces.

In figures $7 \mathrm{a}$ and $7 \mathrm{~b}$ the particle tracking of copolymer microspheres is done using a plug-in written for Java based image processing programm, Image J [26]. In figure 7a the shape of sessile droplet can be clearly recognized, but in figure $7 \mathrm{~b}$ the sessile droplet on the hydrophobic surface seems to be cropped from the left and right sides. This is due to the high contact angle of the sessile droplet. The OCT can not visualize the triple contact line if the drop has a contact angle larger than $90^{\circ}$. The white line is the interface line between glass and air. The droplet lies on the glass on the top part of the interface line (see Fig. 9) and the extension below the surface is due to the refraction of the ray through the liquid.

In our experiments, the flow circulations in the droplet can be observed easily until the skin formation on the surface reaches a certain thickness. As soon as the skin gets thicker, the circulation is suppressed by the skin stress but the final shape is decided by both the flow directions and initial contact angle. Ristenpart et. al. [10] claimed in their paper that the reverse Marangoni 
flow is possible to observe if the temperature gradient is negative, which might be realized by using organic liquids on insulating surfaces. Moreover they supported their theory by changing the contact angle of the sessile drop. In our case we observed a reverse flow (inward flow) by changing the hydrophobicity of the surface.

a)

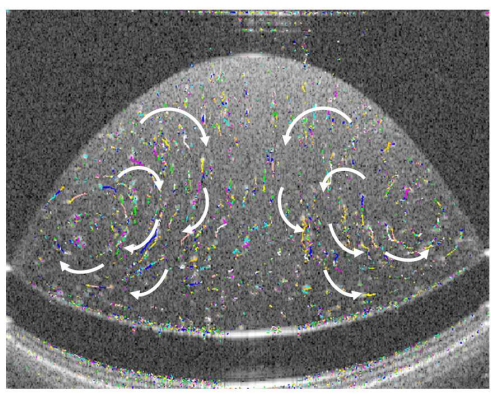

b)

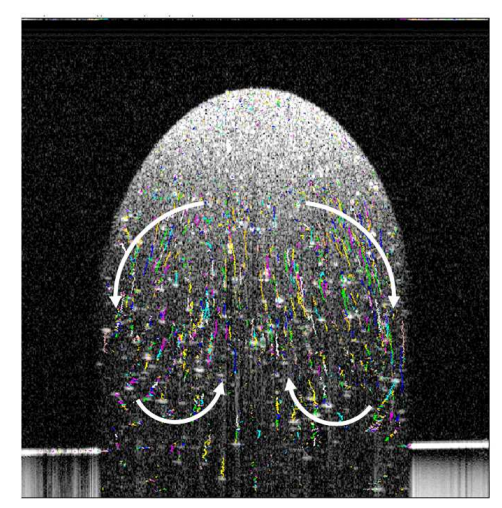

Figure 7: a) On a hydrophilic surface the polymer particles flow from the center to the edges of the sessile droplet. b) On a hydrophobic surface the polymer particles flow from the edges to the center of the sessile droplet (OCT images).

In figures 8 and 9 we show the successive snapshots taken by OCT during the desiccation process of two sessile droplets on hydrophilic and hydrophobic surfaces ${ }^{2}$. The last images represent how the particle accumulation within the sessile drop occurs. The cross-sectional view of the dry sessile droplet on the hydrophilic surface exhibits the "bagel" shape, having a bulge at the edges on the contact line and a dip in the center. However, the cross-sectional view of the dry sessile droplet on the hydrophobic surface reveals an unexpected evolution. At the end of the drying process, a hollow gap occurs under the drop skin as the water evaporates. This evolution cannot be detected by a side view image using a regular camera, unless after the drying is complete, the dried sample be picked off and the cavity can be observed from the back side.

OCT measures the optical path length rather than the physical path length ${ }^{3}$. Therefore the depth profiles need to be corrected by the group refractive index of the material. We determined the time dependent refractive index of the polymer dispersion drop following the method of Lawman \& Liang [24] to show the increasing density of the mixture during desiccation. Using this method we also proved that the evolved cavity in the paint drop on hydrophobic surface is consistent with water vapor having the refractive index of 1 . The refractive index is calculated by the ratio of the optical height $\mathrm{h}_{\text {optical }}$ (from top to bottom) of the drop to real height $\mathrm{h}_{\text {real }}$ (from top to the surface) (see Fig. 10). $\mathrm{n}=\mathrm{h}_{\text {optical }} / \mathrm{h}_{\text {real }}$. Considering the refractive index of water $\mathrm{n}_{w}=1.33$, glass $\mathrm{n}_{g}=1.5$ and air $\mathrm{n}_{a}=1$, it is concluded that the drop changes its state from water consistence to glassy state.

In figure 11 relationship between advection and diffusion is given by the Péclet number. The Péclet number is defined as $\mathrm{Pe}=v R / D$, where $v$ is the drying velocity estimated from the height change, $R$ is the drop radius and $D$ is the mass diffusion coefficient due to Einstein-Stokes

\footnotetext{
${ }^{2}$ The related movies can be found online under the supplementary data.

${ }^{3}$ In this work, optical depth is not converted to physical depth for none of the OCT images. The snapshots presented here are raw images received from the software of the device.
} 

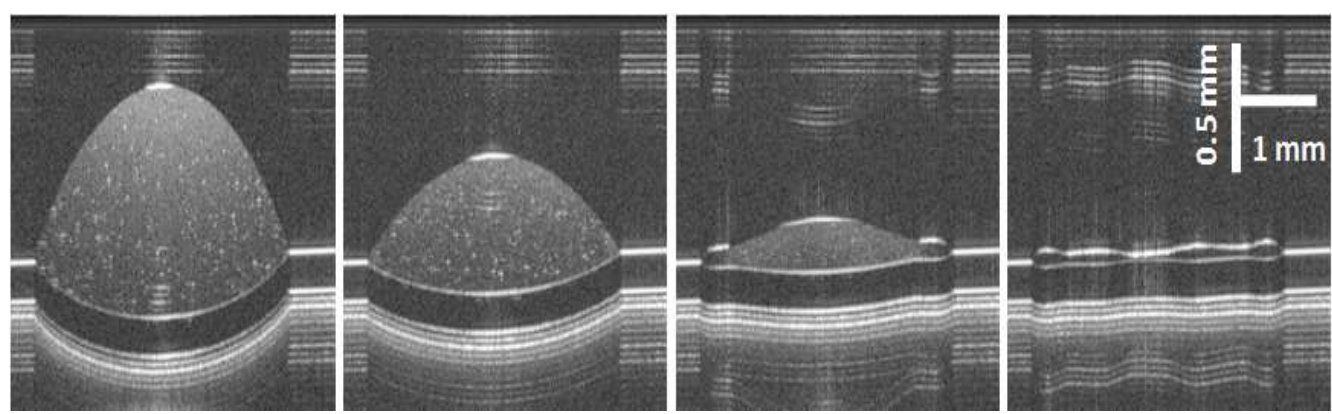

Figure 8: From left to right evaluation snapshots of a drying paint drop on a hydrophilic surface. At the end of the drying process, the polymer particles accumulate at the edges of the contact line, building a donut shape by leaving the center of the drop hollow. The horizontal thin white lines at the top and bottom of the images are artifacts and the transparent layer just below the droplet is the glass slide (OCT images).
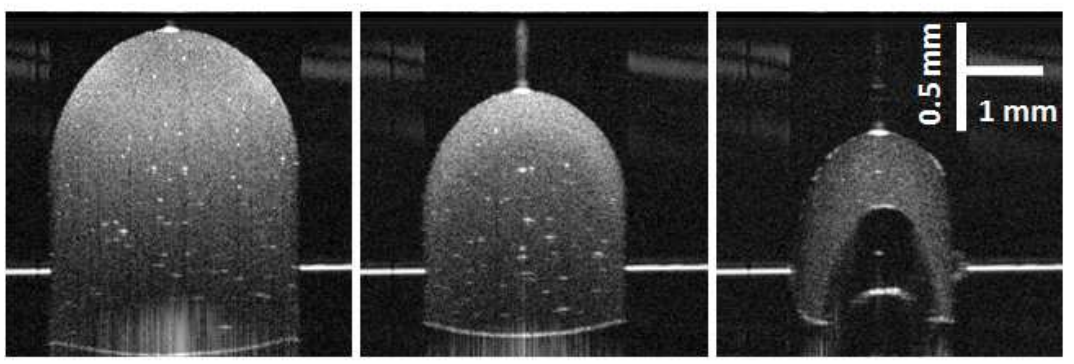

Figure 9: From left to right evaluation snapshots of a drying paint drop on a hydrophobic surface. At the end of the drying process, evaporating water leaves an unexpected cavity beneath the skin which is not predicted before (OCT images).

a)

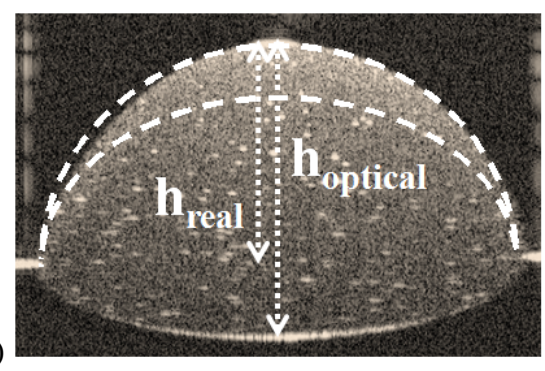

b)

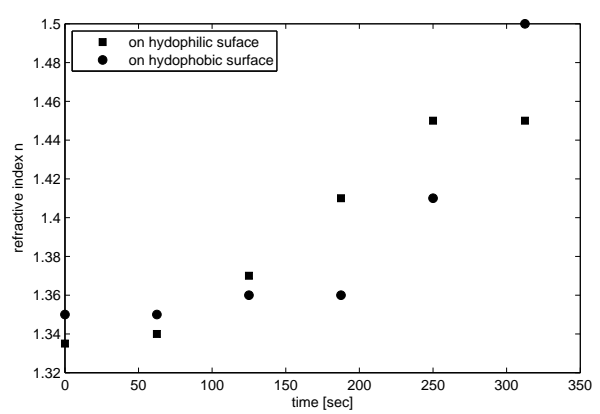

Figure 10: (a) Sessile droplet on a hydrophilic planar surface. The polymer particles accumulate on the surface where the skin is formed and solidified. (b) Refractive index change during the desiccation process. With this method it is shown that the cavity below the dried skin for hydrophobic surface is water vapor $(n=1)$, one of the compounds of the model paint.

equation and is equal to the $\mathrm{D}=k T / 6 \pi \eta r$. Here $\eta$ and $r$ are the viscosity of the fluid and radius of particles in model paint, respectively. High Péclet numbers refer to rapid evaporation [29]. 
From the data in figure 11 we can conclude that sessile polymer drops dry faster on hydrophilic surfaces than on hydrophobic surfaces. This phenomena is related to both the contact area and contact angle. The sessile drop on a hydrophilic surface has a shape of a spherical cap, which has a large contact area and small contact angle. As the drop dries, it gets flatter and takes a form of a thin film. On the other hand, the sessile drop on hydrophobic surface has a smaller contact area due to the compact round shape and a large contact angle. As soon as the skin formation is completed for this case, it becomes difficult for the droplet on a hydrophobic surface to desiccate.

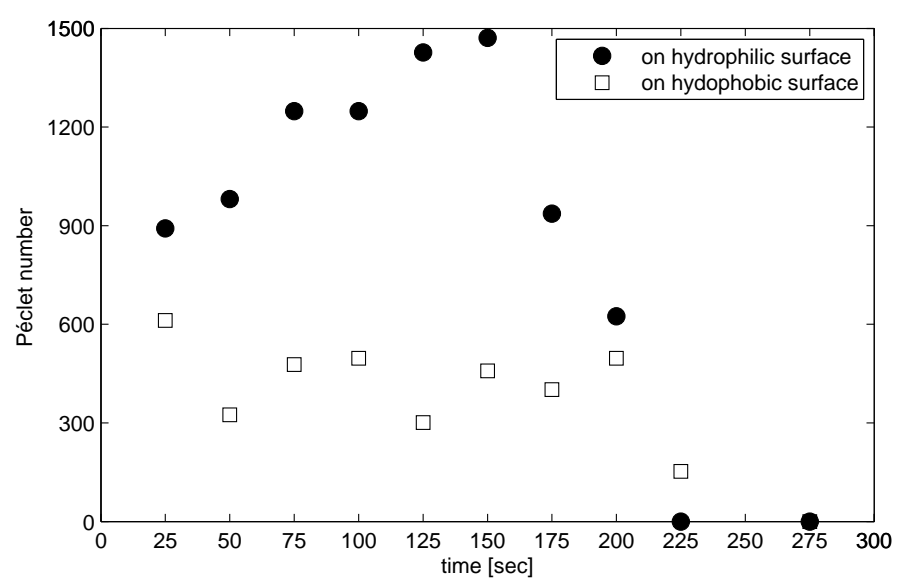

Figure 11: The velocity-time interpretation due to Péclet number for $30 \%$ polymer dispersion-70\% distilled water mixture on both hydrophobic and hydrophilic surfaces. The drop evaporates faster on hydrophilic surfaces.

\section{Discussion}

Regarding the origin of the flow we propose a model that comprises of two assumptions: a solvent evaporation rate controlled by solvent vapor diffusion in the gas phase, and a Marangoni flow essentially associated with the polymer concentration gradients.

Concerning the evaporation rate from the surface of a droplet to the gas phase in the air wedge at the contact line the diffusion-driven steady-state evaporation flow $J_{\mathrm{ev}} \sim-\partial C_{\mathrm{g}} / \partial n$ per unit area, with $\nabla^{2} C_{\mathrm{g}}=0$, is singular at the contact line [3], and $C_{\mathrm{g}}(z) \sim C_{\mathrm{sat}}-b \operatorname{Re}(-\{x+\mathrm{i} y\})^{\frac{\pi}{2 \pi-2 C_{C A}}}$ is the asymptotic solvent concentration in the air wedge at the contact line. The coordinates $(x, y)$ correspond to a projection of a cross section plane to the complex plane, with origin at the contact line, and the $x$ axis being the substrate surface. $\partial / \partial n$ is the derivative normal to the liquid surface, and $C_{\text {sat }}$ the saturation concentration, and $b \geq 0$ some constant. In the air wedge one obtains $J_{\mathrm{ev}} \sim d^{\alpha}$, with $\alpha=\frac{\theta_{C A}-\pi}{2 \pi-\theta_{C A}}$. For hydrophilic surfaces, when $\theta_{C A}<45^{\circ}$, evaporation from the droplet is increased close to the contact line, whereas for hydrophobic surfaces, when $\theta>90^{\circ}$, it is suppressed there. As desiccation proceeds, the polymer concentration at the contact line is raised for hydrophilic, and lowered for hydrophobic substrates as compared to the center of the droplet surface. For our polymer solution this will result in a Marangoni drag, i.e. in a gradient in surface tension oriented in direction of increasing concentration. From figure 5 we estimate that $\sigma_{C}=(\partial \sigma / \partial C)_{T} \sim-0.3 \mathrm{mN} / \mathrm{m} \cdot$ wt.- $\%$, where $\sigma_{C}$ is the change of surface tension with concentration. 
In our OCT records we indeed found substantial differences in polymer concentration, and we therefore assume that this effect is predominant compared to a thermally induced Marangoni flow. We can estimate the gradient of the relative increment of concentration from the gradient of evaporation flow density from mass conservation arguments as $\partial \log C / \partial s \sim \partial \log J_{\mathrm{ev}} / \partial s \sim \alpha s^{-1}$.

Marangoni drag may result in the observed circulation flow. Assuming Stokes equation $0=$ $\eta \nabla^{2} \vec{u}+\vec{\nabla} p$ for the flow velocity $\vec{u}(\vec{r})$, where $\eta$ is the viscosity, and $p \sim \sigma / R$ is the Laplacian pressure, Marangoni drag enters via the boundary conditions at the liquid-gas interface [28]:

$$
\left.\eta \frac{\partial u_{s}}{\partial r}\right|_{\text {surf }}=\frac{\partial \sigma}{\partial s}
$$

$u_{s}$ and $\partial / \partial s$ denote the velocity and derivative tangential to the interface, with solution

$$
u_{s}(r) \approx u_{s}(0)+\frac{\sigma_{C} r^{2}}{2 \eta R} \frac{\partial C}{\partial s}
$$

where $R$ is the drop radius, $r \leq R$ is denoting a shell inside the drop, and $u_{s}(0)$ is a constant that accounts for the back flow of liquid in the center of the drop. The difference in flow velocity between surface and center of the drop is therefore

$$
u_{s}(R)-u_{s}(0) \sim \frac{\sigma_{C} C \alpha}{2 \eta}=\frac{\sigma_{C} C}{\eta} \frac{\theta_{C A}-\pi}{2 \pi-2 \theta_{C A}} .
$$
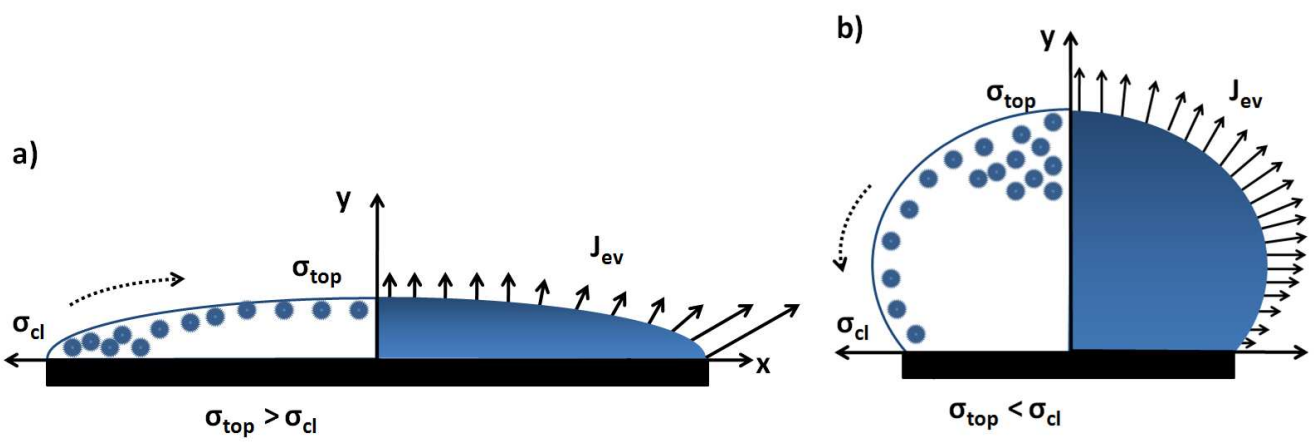

Figure 12: The sketch of evaporation profile and part accumulation of sessile drops resting on hydrophilic (a) and hydrophobic (b) surfaces. The particle accumulation causes a surface tension change which promote a Marangoni circulation. The length of arrows indicate the strength of evaporation rate. The dashed arrow shows the direction of surface tension driven Marangoni flow.

Flow direction is directly related to the wetting angle $\theta_{C A}$, and it is opposite for hydrophilic and hydrophobic substrates. For $\sigma_{C}<0$, as in the present case, the flow velocity at the surface is oriented towards decreasing concentration. For hydrophilic substrates $\left(\theta<45^{\circ}\right)$ this means that the flow at the interface is directed away from, for hydrophobic surfaces $\left(\theta>90^{\circ}\right)$ it is oriented towards the contact line. This is precisely what our OCT experiments are showing. With the observed values for the surface tension gradients, fluid viscosity, and contact angle we obtain an estimation for flow velocities of order of $0.1 \mathrm{~mm} / \mathrm{s}$ which is roughly compatible with 
our observations. We emphasize that this is related to the early stages of desiccation, and that the situation may be different in later stages due to elastic surface stresses resulting from skin formation.

\section{Conclusion}

With this work we have shown how OCT offers an alternative flow visualization method to confocal microscopy, which is predominantly used in literature for such studies. For turbid and barely transparent liquids OCT is an excellent imaging method. It offers an easier and practical method compared to conventional confocal microscopy visualization, which enables observation of the cross-sectional area of the standing liquid without disturbing the process. The OCT method allows us to visualize the flow circulation directions in sessile polymer dispersion droplets on hydrophobic and hydrophilic surfaces. Depending on the contact angle, the final shape of the sessile polymer dispersion drop corresponding to the lowest energy is either a peak or an inversion of curvature leading to the formation of a hump on the axis of the droplet. Under the present conditions only axisymmetric distortions were observed. The flow inside the drop and the initial contact angle decide the drop's final shape. On hydrophobic surfaces (initial contact angle larger than $90^{\circ}$ ) the dispersed particles in the droplet agglomerate in the axis and build a hump due to convection from the contact line to the center. On hydrophilic surfaces (initial contact angle smaller than $45^{\circ}$ ) the dispersed particles in the droplet agglomerate on the contact line and build a bagel due to convection from center to the contact line.

Despite their different structures and physicochemical properties, the same type of shape instabilities on hydrophobic and hydrophilic surfaces have been observed in PEG (polyethylenglycol) solutions and some commercial paint samples (such as nail polisher). A study of mechanical buckling instabilities in dispersion systems may provide clues to solve a variety of problems related to coating processes and painting applications.

Acknowledgement. The authors would like to thank to Rebecca Lange from Nottingham Trent University for providing help for ImageJ applications. This work is financially supported by EXC 256 Center of Smart Interfaces.

\section{Supporting Information}

\subsection{The model paint formulation}

\begin{tabular}{lll} 
Material & $\%$ & additional information \\
\hline \hline Neocryl XK 232 & 77.1 & (Binder, DSM NeoResins) \\
\hline Ammonia to pH 8.5 & 2.8 & \\
\hline Diethylene glycol monobutyl ether & 1.9 & (Co-solvent, Butyl Carbitol, Dow) \\
\hline Thixol 53 L/Water 1:10 & 9.8 & (Acrylate thickener, CoAtex) \\
\hline Borchigel L 75 N/Water 1:1 & 0.4 & (PU thickener, Borchers /Lanxess) \\
\hline Water & 8.0 & \\
\hline
\end{tabular}

\subsection{OCT Imaging of Drying Water Droplet with Particles Inside}

In figure 13 the drying drops of the suspensions of large chemically inert polystyrene polymer microspheres in water are shown. The polymer density is $1.05 \mathrm{~g} / \mathrm{cm}^{3}$ and the index of refraction is 1.59 at $589 \mathrm{~nm}$. They are packaged as aqueous suspensions at $10 \%$ solids by weight. This 
observation with OCT gave us the evidence that pinning of contact line and the surface features orient the accumulation of the particles in the suspension. The cross sectional imaging shows evaluation of the famous coffee ring effect realized by water/particle suspension. Here on hydrophilic surface, the particles accumulate at the contact line and on hydrophobic surface in the center. In both cases, the contact line is pinned due to the surface features. It is concluded that contact angle and pinning affects the evaporation crucially.

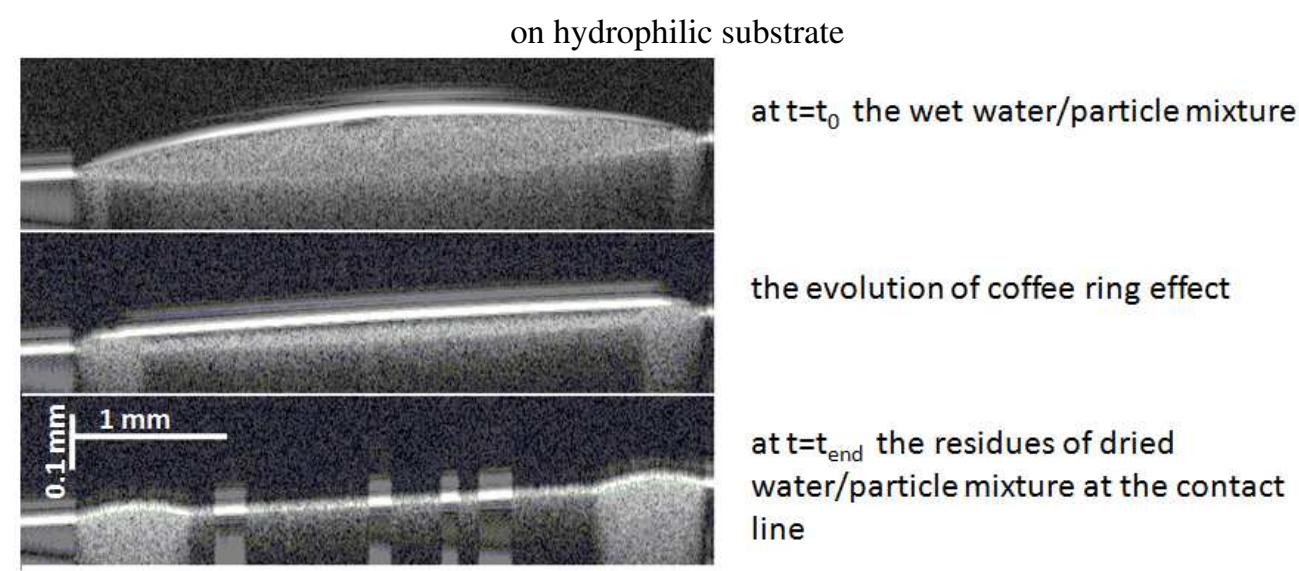

on hydrophobic substrate

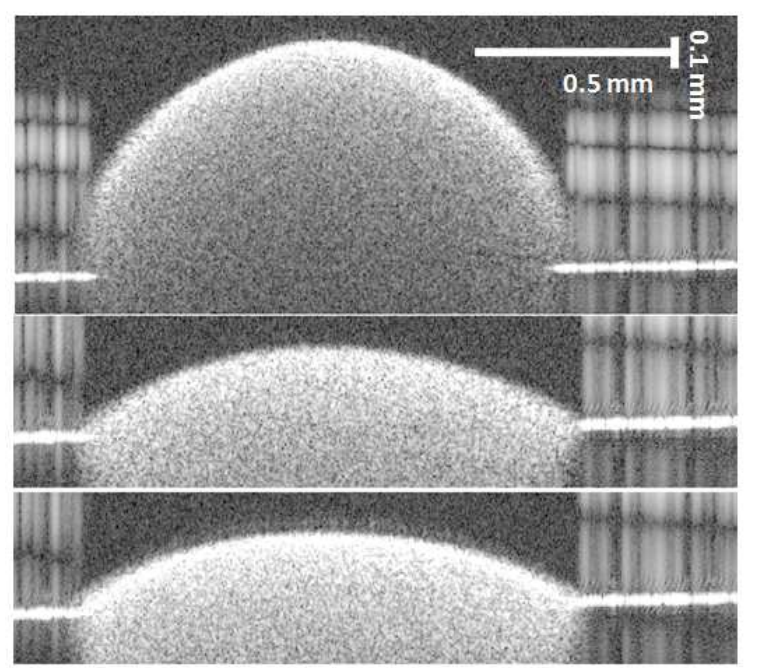

at $\mathrm{t}=\mathrm{t}_{0}$ the wet water/particle mixture

the evolution of anti-coffee ring effect

at $\mathrm{t}=\mathrm{t}_{\text {end }}$ the residues of dried water/particle mixture in the center

Figure 13: The water-polystyrene microsphere suspension dries on hydrophilic and hydrophobic surfaces by gathering the particles at the contact line and in the center respectively .

Supporting Data. The video recordings of OCT images are available online under supporting data. 


\section{References}

[1] R.D. Deegan, O. Bakajin, T.F. Dupont, G. Huber, S.R. Nagel, T.A. Witten, Nature $3891997827-829$.

[2] R.D. Deegan, Physical Review E 61(1) 2000475.

[3] R.D. Deegan, O. Bakajin, T.F. Dupont, G. Huber, S.R. Nagel, T.A. Witten, Physical Review E $62(1) 2000756$.

[4] Y. Morozumi, H. Ishizuka, J.J. Fukai, Chem. Eng. Jpn. 37 (6) 2004778784.

[5] J. Park, J. Moon, Langmuir 222006 3506-3513.

[6] J. Fukai, H.Ishizuka, Y. Sakai, M. Kaneda, M. Morita, A. Takahara, International Journal of Heat and Mass Transfer $4920063561-3567$.

[7] H. Hu, R. G. Larson, Journal of Physical Chemistry B, 1102006 7090-7094.

[8] C. Pauchard, C. Allain, Europhys. Lett. 62(6) 2003 897-903.

[9] L. Pauchard, C. Allain, Physical Review E 682003052801.

[10] W.D. Ristenpart, P.G. Kim, C. Domingues, J. Wan, H.A. Stone, Phys. Rev. Lett. 992007234502.

[11] Y.O. Popov, Physical Review E 712005036313.

[12] D.A. Head, J. Phys.: Condens. Matter 182006485.

[13] R. Bhardwaj, X. Fang, P. Somasundaran, D. Attinger, Langmuir 26(11) 201078337842

[14] R. Bhardwaj, X. Fang, D. Attinger, New Journal of Physics 112009075020

[15] K.L. Maki, S. Kumar, Langmuir 272011 11347-11363.

[16] H. Bodiguel, J. Leng, Soft Matter 620105451.

[17] C. Poulard, P. Damman, EPL Journal 80200764001.

[18] I.G. Marin, H. Gelderblom, D. Lohse, J.H. Snoeijer, PRL 1072011085502.

[19] A.D. Nikolov, D.T. Wasan, Ind. Eng. Chem. Res. 4820092320.

[20] K.H. Kang, S.J. Lee , C.M. Lee , I.K. Kang, Meas. Sci. Technol. 1520041140.

[21] P.J. Jaijus, A. Singh, AIChE Journal 5620091674.

[22] W. Drexler, J.G. Fujimoto, Optical Coherence Tomography: Technology and Applications, Springer 2008.

[23] D. Stifter, Appl. Phys. B 882007337357.

[24] S. Lawman, H. Liang Applied Optics 5020116039.

[25] Thorlabs Spectral Radial SR-OCT, Operating Manual www.thorlabs.com

[26] I. F. Sbalzarini, P. Koumoutsakos J. Struct. Biol. 151(2) 2005 182-195.

[27] T. Kajiya, W. Kobayashi, T. Okuzono, M.J. Doi, Phys. Chem. B. 47200915460.

[28] T. G. Myers, Proceedings of the First Biennial European Coating Symposium, Leeds U.K., World Scientific 1996 , ISBN 9810225431

[29] K. A. Baldwin, S. Roest, D. J. Fairhurst, K. Sefiane, M. E. R. Shanahan, J. Fluid Mech. 695 2012 321-329. 